\title{
Factors influencing compliance to the infection control precautions among nurses and physicians in Jordan: A cross-sectional study
}

Journal of Infection Prevention 2017, Vol. 18(4) 182-188 (C) The Author(s) 2017 Reprints and permissions: sagepub.co.uk/journalsPermissions.nav DOI: $10.1|77 / 1757| 774 \mid 7693676$ jip.sagepub.com @SAGE

\author{
Maysa Nofal', Maha Subih ${ }^{2}$ and Mahmoud Al-Kalaldeh²
}

\begin{abstract}
Background: Adherence to infection control precautions (ICP) is important to reduce the transmission of healthcareassociated infections (HAls).

Aims: To determine nurses and physician's knowledge, attitude and compliance to ICPs and factors associated with reported compliance.

Methods: A cross-sectional survey of nurses and physicians recruited from three hospitals at three different healthcare sectors in Jordan. Three instruments were used to assess knowledge, attitudes and compliance to ICPs.

Findings: A total of 21 I professionals completed the survey: I55 nurses and 56 physicians. Both groups had low knowledge scores for ICP but a high positive attitude. Although both groups had high reported compliance scores, nurses scores were higher $(P=0.04)$. Participants from the private hospital had higher knowledge and compliance scores. Length of experience, knowledge and attitude were significant predictors of reported compliance to ICPs.

Discussion: Despite poor knowledge, Jordanian healthcare professionals reported high scores for positive attitudes and compliance with IPCs. Clinical training programmes are required to enhance knowledge and understanding of IPCs.
\end{abstract}

Keywords

attitude, compliance, infection control precautions, knowledge

Date received: 22 April 2016; accepted: 8 January 2017

\section{Background}

Healthcare-associated infections (HAIs) remain a serious unwanted adverse effect increasing morbidity and mortality of hospital patients and increasing healthcare costs (Defez et al., 2008; Liata et al., 2009). HAIs increases the cost of healthcare including medications, supplies, diagnostic studies and the length of hospital stay (Pittet and Donaldson, 2006; Siegel et al., 2007). One of the greatest challenges is that precautions are available but not applied (Gammon et al., 2008; Pittet, 2001). Interventions to improve adherence to standard infection control precautions (ICPs) should incorporate ongoing evaluation of barriers impeding the successful implementations of these guidelines (Askarian et al., 2005). It is known that improving knowledge and attitudes of healthcare professionals (HCPs) to infection control standards may influence their compliance with recommended practice (Aboelela et al., 2007). In addition, understanding the knowledge and attitudes can help to target educational strategies in order to improve HCPs' practice considering their perspectives and needs (Askarian et al, 2005, Paudyal et al., 2008).

'Faculty of Nursing, University of Jordan, Amman, Jordan

${ }^{2}$ Faculty of Nursing, Al-Zaytoonah University of Jordan, Amman, Jordan

Corresponding author:

Mahmoud Al-Kalaldeh, Faculty of Nursing, Al-Zaytoonah University of Jordan, Amman, P.O. Box 694-I I592, Jordan.

Email: kalaldeh82@yahoo.com 
The existing evidence about the HCPs' behaviours towards ICPs does not provide an in-depth insight about the factors influencing compliance to these precautions worldwide (Pittet, 2001; Boyce and Pitte, 2002; Gammon et al., 2008). Because Jordan is one of the countries with few studies in this field of practice, this study aimed to examine Jordanian nurses' and physician's knowledge, attitude and compliance to standard precautions and factors associated with compliance to ICPs.

\section{Methods}

A descriptive, cross-sectional, correlational design was used to assess both nurses' and physicians' perspectives about ICPs in Jordan. One hospital from each healthcare sector in Jordan, government (general hospitals), private and teaching centres, were selected based on predetermined criteria which recommended the hospital to be referral, and with a capacity of no fewer than 100 beds. Participants were recruited from two strata: registered nurses and physicians. Eligible participants were those who had clinical experience of more than one year, had a bedside clinical duty and were able to speak English. Other administrative personnel who did not have a bedside care role were excluded from the study. To calculate the difference between study groups (physicians versus nurses) accurately, statistical power should be carefully adjusted to determine the required sample size. It was assumed that the sample size should meet the power of 0.80 at medium effect size and an alpha significance level of 0.05 . G*Power is a popular web-based tool used to compute statistical power analyses for many different statistics in psychology such as $t$ tests, $F$ tests, $\chi^{2}$ tests (Polit and Beck, 2008). Using this statistical procedure and based on the earlier assumptions, the required sample size for this study was 160 participants. However, a total of 300 questionnaires were distributed in all sectors (100 for each sector) to account for possible attrition.

\section{Instruments}

The study questionnaire included four parts. The first part was sociodemographic and included questions related to age, gender, marital status, job title, educational level, place of work, length of experience and previous training on infection control.

The second part measured the knowledge of infection control. This tool was developed by a previous study conducted in Jordan (Darawad and Al-Hussami, 2013) to assess knowledge of infection control. The whole questionnaire was derived from the previous study without any change. This tool included 24 multiple choice questions and each question had four alternative choices with ' 1 ' for a correct answer and ' 0 ' for an incorrect answer. The total knowledge score was 24: scores in the range of 20-24 indicate higher knowledge; scores in the range of 14-19 indicate moderate knowledge; and scores less than 13 indicate a low level of knowledge.

The third part of the questionnaire measured the attitude of HCPs towards infection control. This tool was developed by Chan et al. (2007) and contained three sub-scales: the first sub-scale measures the attitude of HCPs towards choosing personal protective equipment (PPE); the second sub-scale measures the attitudes of HCPs towards wearing PPE; and the third sub-scale measures the attitude of HCPs towards high-risk procedures. This tool consists of 11 questions scored using a Likert scale in which 1 corresponds to 'strongly disagree' and 5 to 'strongly agree'. The total attitude scores were clustered into positive attitudes, which were in the range of 34-55, and negative attitudes, which were in the range of 11-33. The reliability of this tool was reported by the previous study as above 0.70 using Cronbach's alpha.

The fourth part of the study questionnaire measured the reported compliance of HCPs to infection control. This was conducted using a specific compliance tool developed by a previous study (Chan et al., 2007). This tool consists of 15 items scored from 1 'never' to 4 'always'. This tool examines the adherence to PPE, disposal of sharps and waste products, decontamination and prevention of cross-infection between patients. The total compliance scores were divided into 'low compliance' in the range of 15-30, 'moderate compliance' in the range of 31-45, and 'high compliance' in the range of 46-60. The reliability test using Cronbach's alpha statistic was 0.76 (Chan et al., 2007).

The study questionnaire has been subject to content validation achieved through seeking the opinions of two expert researchers in this field in addition to two infection control specialists from Jordan University Hospital. The content validity index (CVI) revealed high inter-rater agreement at 0.90. The study questionnaire was provided in English, assuming that the majority of nursing and medical staff have a good command of English as it is the main language for communicating and documenting patient care in hospitals. A pilot study was conducted on 20 nurses and physicians to ensure the clarity of the instruments.

\section{Data collection}

Approvals were obtained from the Scientific and Ethical Committee at the Faculty of Nursing at the University of Jordan and from ethical committees at each participating hospital. Eligible participants from nurses were identified by the coordination with the nursing manager of each hospital who provided a list of all participants meeting the inclusion criteria. Likewise, eligible doctors were identified by the head of medical residents based in each hospital. All eligible participants were invited by sending them a package of papers included the information sheet, the informed consent and the study questionnaire. The information sheet contained the invitation of the study and all related information about the study including ethical issues 
regarding anonymity and confidentiality. Participants were asked to return the completed questionnaires to the head of the department in a sealed envelope (provided) within one week from submission date. Data collection was carried out in the period between June 2013 and January 2014.

\section{Data analysis}

Data were entered into the statistical package for social sciences software (SPSS, Chicago, IL, USA, version 17). A preliminary screening was carried out for missing data and outliers. Descriptive statistics included the calculation of arithmetic mean, standard deviation and frequency. The independent $t$ test was used to examine differences between physicians and nurses in regard to knowledge, attitude and compliance scores. In addition, the one-way ANOVA test was used to examine differences between participants from different healthcare sectors. Pearson Correlation Coefficient was used to examine the relationship between study variables and their relationships with demographical variables. These significant variables were further examined using a regression model. Multiple hierarchical regression was conducted to determine predictors that influence the compliance to ICPs. Using this statistical procedure, each possible predictor was entered separately into blocks to examine the impact of each factor on the overall model based on the adjusted $\mathrm{R}^{2}$. Variables entered to the models were age, knowledge, experience and attitude.

\section{Results}

Of 300 distributed questionnaires, 211 were completed and retained, giving a $70.3 \%$ response rate. Of those, 155 $(73.5 \%)$ were nurses and $56(26.5 \%)$ were physicians. Participants' demographics are shown in Table 1. Participants were distributed evenly over the three hospitals. The majority of nurses were women $(56.8 \%)$ while the majority of physicians were men $(64.3 \%)$. Physicians were slightly older than nurses while nurses had slightly longer clinical experience. Regarding working units, most of the nurses were in the medical, surgical and the intensive care units and about $25 \%$ were in the day case and delivery rooms. Likewise, most physicians were in medical, surgical and paediatric units. The majority of nurses (56.8\%) attended a training course about the ICPs while fewer physicians $(32.1 \%)$ have attended such a course. However, the majority of both professionals declared their desire to enrol ICPs training program (Table 1).

\section{Knowledge, attitudes and compliance to ICPs}

Nurses and physicians were compared with regard to the total knowledge, attitudes and compliance scores. As shown in Table 2, the mean score of each variable was calculated. Regarding knowledge of ICPs, both nurses and physicians revealed knowledge scores less than 13 which are considered low levels of knowledge, showing no significant difference between both groups. However, both nurses and physicians scored higher in having positive attitudes toward ICPs (scores were above 33), and there was no statistical difference found between both groups as shown in the table. In relation to compliance scores, nurses scored higher compliance to ICPs compared with physicians $(P=0.04)$, although both reported high level of compliance based on their scores which were above 45 (Table 2). However, this difference in compliance between physicians and nurses is likely confounded by gender, as women are more likely to comply with infection control guidelines more than male participants (Gammon et al., 2008).

Testing mean difference of the reported behaviours regarding knowledge, attitude and compliance towards ICPs between the three hospitals was performed using oneway ANOVA test. The results showed that there was a statistically significant difference between the three types of hospitals regarding level of knowledge $(F=14.43$, df $=$ 208, $P<0.001)$. A Tukey's HSD post-hoc test revealed that the private hospital had the highest knowledge level $(13 \pm$ 2.8, $P<0.05)$ compared to the governmental and teaching hospitals which had lower knowledge scores $(10.6 \pm 3.1$, $P \leq 0.05 ; 12.5 \pm 2.9$, respectively).

Also, there was a statistical significant difference between these hospitals in respect to compliance scores $(\mathrm{F}=8.63, \mathrm{df}=208, P<0.001)$. Using Tukey's HSD post hoc test, the compliance scores of the private hospital $(\mathrm{M}=$ $51.2 \pm 6.7)$ appeared significantly higher than both governmental and teaching hospitals $(\mathrm{M}=46.5 \pm 5.7$ and $\mathrm{M}=48.7$ \pm 7 , respectively). Regarding attitudes scores, there was no statistical difference between the three hospitals with regard to attitudes scores $(\mathrm{F}=1.8, \mathrm{df}=208, P=0.16)$.

\section{Factors influencing compliance to ICPS}

Initially, Pearson's correlation was conducted to show the direction and strength between compliance and other related variables. After examining the correlations between all variables under study, it was shown that there was a significant positive correlation between compliance and attitude $(\mathrm{r}=0.36, P<0.01)$ and between compliance and knowledge $(\mathrm{r}=0.31, P<0.01)$. In addition, the result showed a significant positive correlation between compliance and both age and length of experience $(\mathrm{r}=0.28$, $P \leq 0.01, \mathrm{r}=0.32, P \leq 0.01$, respectively).

Hierarchical multiple regression analysis was used to examine independent predictors that significantly affect compliance with ICPs (Table 3). These variables which showed significant positive correlations were entered individually into blocks. The first block which contained the age variable revealed that $8.7 \%$ of the variance of 
Table I. Participants' demographics.

\begin{tabular}{|c|c|c|c|c|}
\hline Variable & Physicians $(n=56)$ & Nurses $(n=155)$ & Total $(\mathrm{N}=2 \mathrm{I} \mathrm{I})$ & \\
\hline Hospital & n (\%) & n (\%) & n (\%) & Sig. \\
\hline Governmental & $24(42.9)$ & $57(36.8)$ & $81(38.4)$ & 0.845 \\
\hline Private & $15(26.8)$ & $40(25.8)$ & $55(26.1)$ & \\
\hline Teaching & $17(30.4)$ & $58(37.4)$ & $75(35.5)$ & \\
\hline \multicolumn{5}{|l|}{ Gender } \\
\hline Male & $36(64.3)$ & $67(43.2)$ & $103(48.8)$ & 0.076 \\
\hline Female & $20(35.7)$ & $88(56.8)$ & $108(5 \mid .2)$ & \\
\hline Age (years), mean (SD) & $32.5(6.5)$ & $28.8(5.8)$ & $30.65(11.3)$ & 0.205 \\
\hline Clinical experience (years), mean (SD) & $3.4(1.6)$ & $5.1(4.3)$ & $4.25(2.5)$ & 0.126 \\
\hline \multicolumn{5}{|l|}{ Working area } \\
\hline Medical ward & $15(26.8)$ & $42(27.1)$ & $57(27.3)$ & 0.082 \\
\hline Surgical ward & $16(28.5)$ & $19(12.2)$ & $35(16.8)$ & \\
\hline Critical care units & $6(10.8)$ & $22(14.2)$ & $28(13.3)$ & \\
\hline Paediatric ward & $13(23.3)$ & $15(9.7)$ & $28(13.3)$ & \\
\hline Emergency department & $5(8.9)$ & $18(11.6)$ & $23(10.9)$ & \\
\hline Others (e.g. day case and delivery rooms) & $\mathrm{I}(\mathrm{I} .7)$ & $39(25.2)$ & $39(21.4)$ & \\
\hline \multicolumn{5}{|l|}{ Has attended any training course on ICPs } \\
\hline Yes & $18(32.1)$ & $88(56.8)$ & $106(50.2)$ & 0.091 \\
\hline No & $38(67.9)$ & $67(43.2)$ & $105(49.8)$ & \\
\hline \multicolumn{5}{|l|}{ Need to enrol in a training course on ICPs } \\
\hline Yes & $45(80.4)$ & I $33(85.8)$ & $178(84.4)$ & 0.006 \\
\hline No & II (19.6) & $22(14.2)$ & $33(15.6)$ & \\
\hline
\end{tabular}

Sig. level was tested by Wilcoxon-Mann-Whitney test for continuous variables and by Fisher's exact test for categorical variables. $\mathrm{N}$, sample size; $\mathrm{n}$, frequency.

Table 2. Comparisons between nurses and physicians on knowledge, attitude and compliance to ICPs.

\begin{tabular}{|c|c|c|c|c|c|}
\hline \multirow[b]{2}{*}{ Variable } & \multirow{2}{*}{$\frac{\text { Nurses }}{\text { Mean (SD) }}$} & \multirow{2}{*}{$\frac{\text { Physicians }}{\text { Mean (SD) }}$} & \multicolumn{3}{|c|}{ Students t-test } \\
\hline & & & $\mathrm{df}$ & $\mathrm{t}$ & Sig. \\
\hline Knowledge & $12.2(2.8)$ & 11.9 (3.3) & 209 & 0.70 & 0.48 \\
\hline Attitude & $38.3(6.8)$ & 38.7 (7.6) & 201 & 0.26 & 0.78 \\
\hline Compliance & 49.1 (6.7) & $46.9(6.5)$ & 201 & -2.05 & 0.04 \\
\hline
\end{tabular}

Significant level was set at 0.05 (two-tailed).

$\mathrm{df}$, degree of freedom; SD, standard deviation; $t$, t-statistic.

compliance was explained by age (based on the adjusted $\mathrm{R}^{2}$ ). After entering the length of clinical experience in the second block, the effect was increased to $9.4 \%$ but it was not statistically significant $(P=0.13)$. The third block added to the model was the knowledge scores which influenced the variance of compliance by $16 \%$ based on the adjusted $\mathrm{R}^{2}(P<0.001)$. The fourth block contained the attitudes variable and this increased the effect on compliance to $25 \%(P<0.001)$. Since age and length of clinical experience are linked, it is likely that the latter is more relevant in explaining this relationship. Therefore, the results suggested that clinical experience, knowledge and attitude were significant predictors of compliance to ICPs (Table 3). 
Table 3. Predicting factors influencing compliance using multiple hierarchical regression.

\begin{tabular}{|c|c|c|c|c|c|c|c|c|}
\hline \multirow[b]{2}{*}{ Variables } & \multicolumn{2}{|c|}{ Model I } & \multicolumn{2}{|c|}{ Model 2} & \multicolumn{2}{|c|}{ Model 3} & \multicolumn{2}{|c|}{ Model 4} \\
\hline & $\beta$ & $P$ value & $\beta$ & $P$ value & $\beta$ & $P$ value & $\beta$ & $P$ value \\
\hline Age & 0.30 & $<0.001$ & 0.123 & 0.37 & 0.10 & 0.41 & 0.10 & 0.41 \\
\hline Experience & & & 0.209 & 0.133 & 0.19 & 0.13 & 0.22 & 0.77 \\
\hline Knowledge & & & & & 0.27 & $<0.001$ & 0.18 & 0.005 \\
\hline Attitude & & & & & & $<0.001$ & 0.317 & $<0.001$ \\
\hline $\mathrm{R}^{2}$ & 0.092 & $<0.001$ & 0.11 & 0.13 & 0.18 & $<0.001$ & 0.27 & $<0.001$ \\
\hline Adjusted $\mathrm{R}^{2}$ & 0.087 & $<0.001$ & 0.049 & 0.13 & 0.16 & $<0.001$ & 0.25 & $<0.001$ \\
\hline $\mathrm{R}^{2}$ change & 0.092 & $<0.001$ & 0.011 & 0.13 & 0.075 & $<0.001$ & 0.093 & $<0.001$ \\
\hline
\end{tabular}

Significant at $\alpha=0.001$ two-tailed test.

\section{Discussion}

This survey demonstrated that both nurses and physicians had a low level of knowledge of ICPs which is congruent with previous studies. For instance, a study by Chan et al. (2007), which was conducted in Hong Kong, found that nurses' knowledge regarding to ICPs was inadequate. Similarly, inadequate knowledge of ICPs was evident among healthcare workers (HCWs) in Nepal in which only $22 \%$ of the HCWs reported accurate knowledge (Timilshina et al., 2011). In contrast, a Jamaican study found that nurses and doctors were knowledgeable about the ICPs (Vaz et al., 2010). However, participants in this study, whether nurses or physicians, emphasised the need to attend training programs on ICPs. This indicates that both professional groups recognised the need to improve their knowledge about ICPs. It was apparent that respondents from the private hospital showed higher knowledge comparing with other hospitals. This result which needs more investigation may reflect differences in institutional policies related to continuous education and performance appraisal of employees.

Both nurses and physicians exhibited equal and positive attitudes toward ICPs. This conforms to previous studies which reported the same positive attitudes, such as a study conducted in Italy (Nobile et al., 2002) and another study conducted in Zambia (Mukwato et al., 2009), while a study in Nepal showed that only $14 \%$ of both nurses and physicians had positive attitude towards ICPs (Paudyal et al., 2008). While no differences were found between hospitals, this indicated that the majority of Jordanian professionals perceived the importance of adherence to the ICPs and agreed on the significance of applying the ICPs in different settings.

Although nurses had significantly higher compliance scores than physicians, both professionals reported high compliance in this study. This compares favourably with other studies which have found much lower reported levels of compliance (Askarian et al., 2005; Paudyal et al., 2008).
Other studies have found that physicians reported less compliance to hand-washing practice (Lipsett and Swoboda, 2001). Gershon et al. (1995) found that despite an educational and interventional programme which led to a significant increase in the total compliance score towards the ICPs, physicians were less compliant with ICPs. While reported compliance was found to be high in this study, it is acknowledged that adherence to ICPs is influenced by staffs' attitude towards the importance of this practice. Thus, it is important to note that the participants' of this study also scored highly on attitudes to IPC.

Other studies have demonstrated the relationships between compliance and attitudes with regard to various precautions. A study by Darawad et al. (2012) found that attitudes positively affected compliance with hand washing. Similar results were found in a Chinese study (Chan et al., 2007) and another Iranian study (Askarian et al., 2005). However, a previous study found no significant relationship between compliance and knowledge of ICPs (Vaz et al., 2010).

In relation to sociodemographic factors, this study indicated that both age and length of clinical experience had a positive correlation with compliance to the ICPs. This is consistent with other studies which have found that years of experience impacted positively on compliance with hand washing (Chan et al., 2002; Suchitra and Lakshmi, 2007). However, Timilshina et al. (2011) found that younger nurses had higher compliance to ICPs than others, which may be explained by more years of experience leading nurses to become more reluctant and resistant to change their practices (Osborne, 2003).

Clinical experience, knowledge and attitudes were significant factors contributing to higher compliance towards ICPs among the HCPs included in this survey. In previous studies conducted in Jordan (Al-Hussami et al., 2011; Darawad et al., 2012), attitudes were the most prominent factors predicting compliance to hand washing while age and length of experience were less relevant. 


\section{Limitations}

While study sample was drawn from only three hospitals located in Amman, in which each healthcare sector was represented by one hospital, it is not possible to generalise findings to all healthcare settings at different geographical locations in Jordan. The reported behaviour does not correlate completely with observations of practitioner behaviour and may contradict with the actual practice or perception. Therefore, utilising additional methodologies might be required to more accurately measure compliance, for example, bedside observation combined with self-reporting.

\section{Conclusions}

Jordanian HCPs showed positive attitudes and high levels of reported compliance with ICPs despite low levels of knowledge. Nurses reported higher compliance to ICPs than physicians. The study affirmed that positive attitude has an influence on compliance with the ICPs. Greater knowledge and positive attitudes together with longer clinical experience predicted greater reported compliance to the ICPs. These results highlight the need for training programs for HCPs on ICPs to enhance knowledge and understanding of the ICPs concepts using updated evidence-based guidelines.

\section{Acknowledgements}

The authors thank all the nurses and physicians who participated in this study as well as all the hospital administrators who facilitated the process of accessing the target population.

\section{Declaration of conflicting interests}

The author(s) declared no potential conflicts of interest with respect to the research, authorship, and/or publication of this article.

\section{Funding}

The author(s) received no financial support for the research, authorship, and/or publication of this article.

\section{Peer review statement}

Not commissioned; blind peer-reviewed.

\section{References}

Aboelela W, Stone P and Larson L. (2007) The effectiveness of bundled behavioral interventions to reduce healthcare associated infections: A systematic review of the literature. Journal of Hospital Infection 66: $101-108$

Al-Hussami MO, Darawad MW and Almhairat I. (2011) Predictors of compliance hand hygiene practice among healthcare professionals. Healthcare Infection 16: 79-84.

Askarian M, Shiraly R and McLaws L. (2005) Knowledge, attitude, and practice of contact precautions among Iranian nurse American Journal of Infection Control 33: 486-488.
Boyce JM and Pittet D; Healthcare Infection Control Practices Advisory Committee; HICPAC/SHEA/APIC/IDSA Hand Hygiene Task Force. (2002) Guideline for hand hygiene in healthcare settings: Recommendations of the Healthcare Infection Control Practices Advisory Committee and the HICPAC/SHEA/APIC/IDSA Hand Hygiene Task Force. Society for Healthcare Epidemiology of America/ Association for Professionals in Infection Control/Infectious Diseases Society of America. Morbidity and Mortality Weekly Report 51: 1-48.

Chan M, Ho A and Day MC. (2007) Investigating the knowledge, attitudes and practice patterns of operating room staff towards standard and transmission based precautions: results of a cluster analysis. Journal of Clinical Nursing 17: 1051-1062.

Chan R, Molassiotis A, Chan E, Chan V, Ho B, Lai CY, Lam P, Shit F and Yiu I. (2002) Nurses' knowledge of and compliance with universal precautions in an acute care hospital. International Journal of Nursing Studies 39: 157-163.

Darawad MW and Al-Hussami M. (2013) Jordanian nursing students' knowledge of, attitudes towards, and compliance with infection control precautions Nurse Education Today 33: 580-583.

Darawad MW, Al-Hussami M, Almhairat I and Al-Sutari M. (2012) Investigating Jordanian nurses' handwashing beliefs, attitudes, and compliance. American Journal of Infection Control 40: 643-647.

Defez C, Fabbro-Peray P, Cazaban M, Boudemaghe T, Sotto A and Daures JP. (2008) Additional direct medical costs of nosocomial infections: estimation from a cohort of patients in a French university hospital. Journal of Hospital Infection 68: 130-136.

Gammon J, Morgan-Samual H and Gould D. (2008) A review of the evidence for suboptimal compliance of healthcare practitioners to standard/universal infection control precautions. Journal of Clinical Nursing 17: 157-167.

Gershon R, Vlahov D, Felknor S, Vesley D, Johnson PC, Delclos GL and Murphy LR. (1995) Compliance with universal precautions among health care workers at three regional hospitals. American Journal of Infection Control 4: 225-236.

Liata E, Gaynes P and Fridkin S. (2009) Measuring the scope and magnitude of hospital-associated infection in the United States: the value of prevalence surveys. Clinical Infectious Diseases 48: 1434-1440.

Lipsett A and Swoboda M. (2001) Hand washing compliance depends on professional status. Surgical Infections 2: 241-245.

Mukwato K, Ngoma M and Maimbowla M. (2009) Compliance with infection prevention guidelines by health care workers at Ronald Ross General Hospital Mufulira District. Medical Journal of Zambia 35: $110-116$.

Nobile A, Montuori P, Diaco E and Villari P. (2002) Healthcare personnel and hand decontamination in intensive care units: Knowledge, attitudes, and behaviour in Italy. Journal of Hospital Infection 51: 226-232.

Osborne S. (2003) Influences on compliance with standard precautions among operating room nurses. American Journal of Infection Control 31: 415-423.

Paudyal P, Simkhada P and Bruce J. (2008) Infection control knowledge, attitude, and practice among Nepalese health care workers. American Journal of Infection Control 36: 595-597.

Pittet D. (2001) Improving adherence to hand hygiene practice: A multidisciplinary approach. Emerging Infectious Diseases 7: 234-240.

Pittet D and Donaldson L. (2006) Challenging the world: patient safety and health care-associated infection. International Journal for Quality in Health Care 18: 4-8.

Polit DF and Beck CT. (2008). Nursing Research: Generating and Assessing Evidence for Nursing Practice. Philadelphia, PA: Wolters Kluwer/ Lippincott Williams \& Wilkins.

Siegel JD, Rhinehart E, Jackson M and Chiarello L and Health Care Infection Control Practices Advisory Committee. (2007) Guideline for isolation precautions: preventing transmission of infectious agents 
in health care settings. American Journal of Infection Control 35: S65-S164.

Suchitra J and Lakshmi N. (2007) Impact of education on knowledge, attitudes and practices among various categories of health care workers on nosocomial infections. Indian Journal of Medical Microbiology 25: 181-187.

Timilshina N, Ansari M and Dayal V. (2011) Risk of infection among primary health workers in the Western Development Region, Nepal: knowledge and compliance. Journal of Infection in Developing Countries 5: 18-22.

Vaz K, McGrowder D, Alexander-Lindo R, Gordon L, Brown P and Irving R. (2010) Knowledge, awareness and compliance with universal precautions among healthcare workers at the University of West Indies, Jamaica. International Journal of Occupational and Environmental Medicine 1: 171-181. 